\title{
Peak expiratory flow rate and the acute chest syndrome in homozygous sickle cell disease
}

\author{
E Frances Bowen, Jonathan G Crowston, Karel De Ceulaer, Graham R Serjeant
}

\begin{abstract}
The peak expiratory flow rate (PEFR) was studied in 20 matched pairs of children with homozygous sickle cell disease with either no episodes or six or more episodes of acute chest syndrome. The pairs were carefully matched for height and a highly significant reduction in PEFR was observed in children with multiple episodes of acute chest syndrome. Lateral and anteroposterior chest diameters and chest circumference correlated with PEFR but did not differ between index and control cases. The most likely cause of the reduced PEFR in children with multiple episodes of acute chest syndrome is an accumulating pulmonary fibrosis that decreases lung compliance.
\end{abstract}

Children with homozygous sickle cell (SS) disease are prone to the acute chest syndrome, the relative risk being four times that in normal controls by the age of 4 years. ${ }^{1}$ The term acute chest syndrome covers a range of acute pulmonary episodes including infection, infarction, and sequestration within the pulmonary capillaries. Adults with SS disease have significant reductions in total lung capacity, vital capacity, forced vital capacity, and ratio of residual volume to total lung capacity. ${ }^{2}{ }^{3}$ Furthermore the diffusing capacity of the alveolar membrane is reduced, ${ }^{2} 4$ the greatest reductions occurring in those with a history of pulmonary complications. $^{2}$ Peak expiratory flow rate (PEFR), which might be expected to fall with decreased lung compliance after pulmonary complications, was normal in a small study of 12 SS children and 12 controls aged 7-19 years. ${ }^{5}$ To determine whether peak expiratory flow rate is affected by a history of pulmonary complications, the PEFR has been studied in 20 matched pairs with SS disease, with and without histories of pulmonary complications.

Medical Research Council Laboratories (Jamaica), University of the West Indies, Kingston 7, Jamaica

E Frances Bowen Jonathan G Crowston Karel De Ceulaer Graham R Serjeant

Correspondence and requests for reprints to:

Professor Serjeant.

Accepted 6 September 1990

\section{Patients and methods}

The patients attended the sickle cell clinic of the University Hospital of the West Indies, Kingston, Jamaica, and were participating in a cohort study of sickle cell disease from birth. Between June 1973 and December 1981, 100000 consecutive normal deliveries at the main government maternity hospital (Victoria Jubilee Hospital) were screened. A total of 314 babies with SS disease were detected and they have been followed up prospectively. Of these 55 have died and 31 have emigrated, leaving 228 children at the study date (December 1989/January 1990) aged from 8-16 years. The diagnosis of SS disease was based on standard critiera.

The acute chest syndrome was defined as acute pulmonary symptoms associated with either clinical signs of parenchymal involvement or pulmonary infiltrates on radiography. The frequency of these events was derived by review of the computerised clinical codings and confirmed by examination of patient records. Children with asthma, bronchitis, and who had suffered a stroke were excluded. If two events were separated by less than four weeks the records were reviewed to distinguish a prolonged single event from two separate events. The number of episodes of acute chest syndrome varied from nil to 16 and 31 subjects had had six episodes or more (fig 1). Of these 31 subjects, three had chronic disease possibly influencing lung function (systemic lupus erythematosus, nephrotic syndrome, and recurrent sepsis) and were excluded and three had defaulted leaving 25 cases. It was possible to match 20 of these index cases with 20 children who had never had acute chest syndrome (control group). Each pair was matched for sex, height, and age (in order of priority).

Height was measured, without shoes, with a Harpenden wall mounted stadiometer or a portable stadiometer (checked against the fixed model) for home visits. Anteroposterior and lateral chest diameters were measured with Harpenden chest calipers in the horizontal plane through the fourth sternocostal junction at the end of normal expiration. Chest circumference was measured at the same level and stage of expiration with a standard tape measure. PEFR was measured with a portable peak flow meter

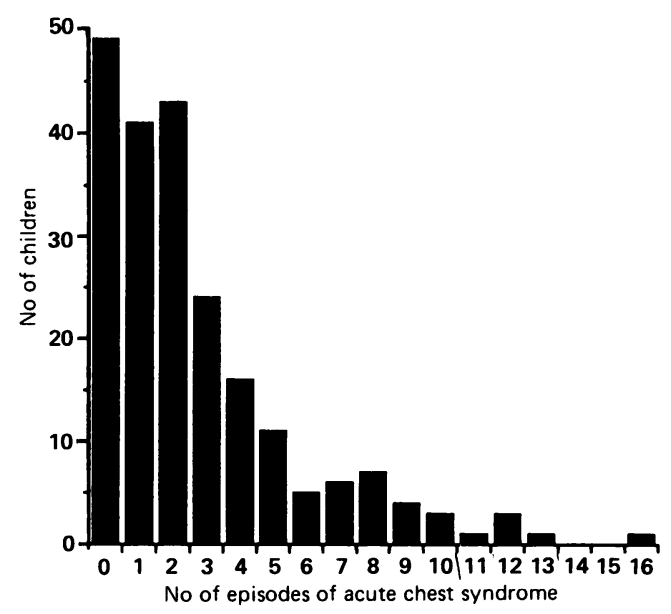

Figure 1 Frequency distribution of episodes of acute chest syndrome in children with SS disease. 
(Mini-Wright, Airmed, Clement-Clarke International) in the standing position and taking the maximum of five successive measurements. The instrument was not independently calibrated but the same instrument was used for the complete study. No other aspect of pulmonary function was measured.

The paired Student's $t$ test was used to compare mean values between the index cases and the controls. Correlations were assessed by the product moment correlation coefficient.

\section{Results}

The mean age of index cases, $12 \cdot 1$ years (range 8-16 years), did not differ from that in controls, 11.6 years (range 8-16). Mean height in index cases, $142 \cdot 4 \mathrm{~cm}$ (range $117 \cdot 5-160 \cdot 0$ ) was similar to that in controls, $143.3 \mathrm{~cm}$ (range 121.0 $169 \cdot 0)$.

\section{CHEST SIZE}

Chest circumference varied from $52-74 \mathrm{~cm}$ but did not differ between index cases (mean (SD) $62 \cdot 0(6 \cdot 2) \mathrm{cm})$ and controls $(63 \cdot 8(5 \cdot 4) \mathrm{cm})$. Chest circumference was significantly correlated ( $r=0.66 \mathrm{p}<0.001$ ) with PEFR (fig 2).

\section{CHEST SHAPE}

Anteroposterior chest diameter varied from $12 \cdot 2-18 \cdot 9 \mathrm{~cm}$ but did not differ between index

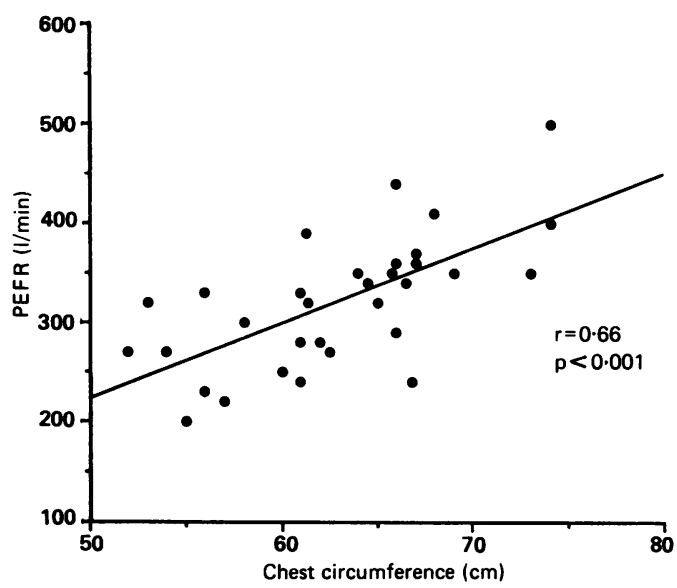

Figure 2 Association between PEFR and chest circumference.

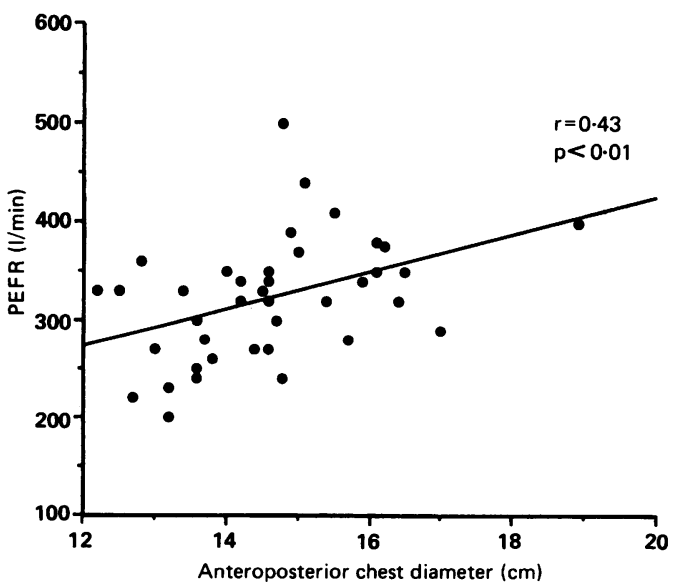

Figure 3 Association between PEFR and anteroposterior chest diameter. cases (mean (SD) $14.7(1.5) \mathrm{cm}$ ) and controls $(14.6(1.3) \mathrm{cm})$. Lateral chest diameters varied from $16 \cdot 6-24 \cdot 6 \mathrm{~cm}$ and also did not vary between index cases $(20 \cdot 2(1 \cdot 8))$ and controls $(20.6(1.9))$. Both measurements correlated with PEFR (figs 3 and 4), although the correlation was closer with lateral diameter $(r=0.69$, $\mathrm{p}<0.001)$ than with anterposterior chest diameter $(r=0.43, p<0.01)$.

The abnormal anthropometry of SS disease affects chest shape by reducing the lateral chest diameter and making a more hoop shaped chest with an increased ratio of anteroposterior to lateral chest diameter. To ascertain whether this abnormal shape determined the abnormalities in PEFR, the association between this ratio and the PEFR was assessed. The ratio did not differ between index cases (mean $1 \cdot 38(0 \cdot 11)$ ) and controls $(1.41(0 \cdot 11))$ and did not correlate with the PEFR $(r=0 \cdot 07, p>0 \cdot 1)$.

PEFR

As expected, the PEFR increased with height in both index cases and controls, ${ }^{6}$ although values were consistently lower in patients with a history of pulmonary complications (fig 5). The difference in PEFR between matched pairs (mean 63.8 (33.4); range $10-120 \mathrm{l} / \mathrm{min}$ ) was significant (paired $t$ test, $\mathrm{t}=8 \cdot 55, \mathrm{p}<0.001$ ). Overall the PEFR in index cases (mean 291 (50);

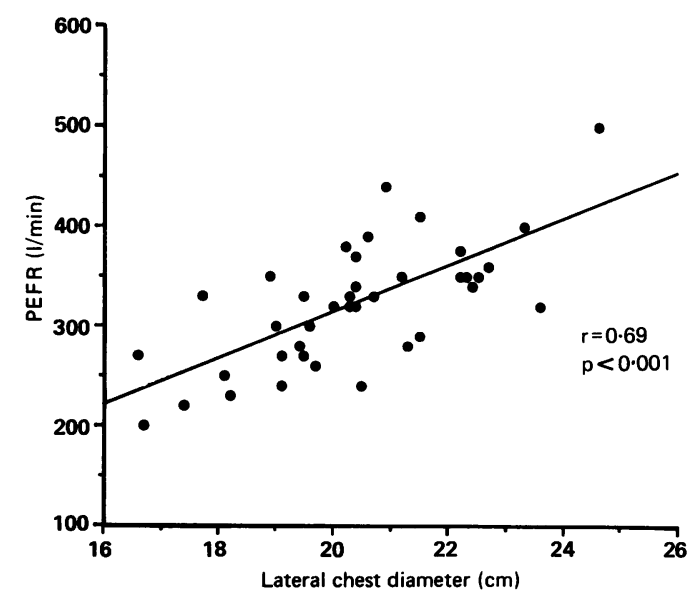

Figure 4 Association between PEFR and lateral chest diameter.

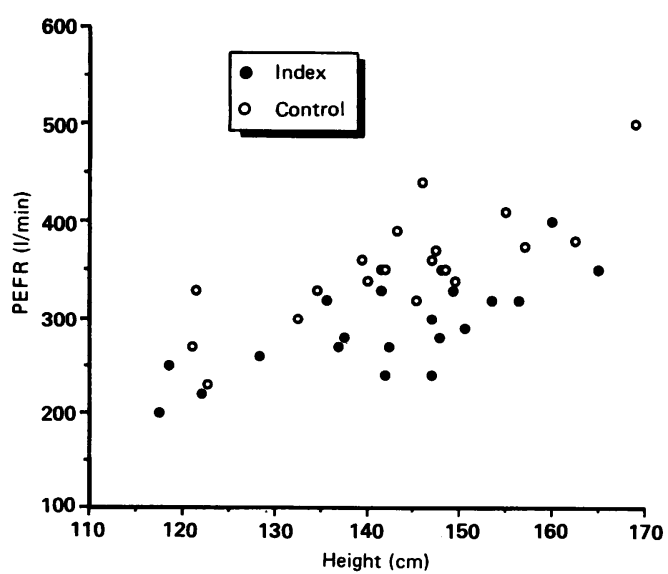

Figure 5 Association between PEFR and height in index and control children. 
range $200-400 \mathrm{l} / \mathrm{min}$ ) was significantly less than in controls (mean 355 (57), 230-500 1/min).

To ascertain whether PEFR showed progressive deterioration with the number or severity of episodes of acute chest syndrome, an analysis of covariance was conducted between PEFR, height, number of episodes of acute chest syndroe or severity of such episodes depicted as the number requiring hospital admission or transfusion treatment. The analysis showed no significant effect on PEFR of either the number or severity of episodes of acute chest syndrome.

\section{Discussion}

Recurrent respiratory infections were noted in the first case report of sickle cell disease ${ }^{7}$ and the susceptibility of children with SS disease to serious pulmonary disease has since been well documented. ${ }^{18}$ Abnormalities of lung volume and function have been reported in SS disease, although it is unclear the extent to which these changes reflect the abnormal chest shape of SS disease or histories of pulmonary complications. An earlier Jamaican study had shown that the diffusing capacity of the alveolar membrane was reduced in SS disease and was decreased further in patients with a history of pulmonary complications. $^{2}$

The PEFR is a simple test of lung function amenable to population studies and domiciliary use. The Mini-Wright peak flow meter is cheap and provides a single reproducible measurement. ${ }^{9}$ The major determinants of PEFR are the static recoil pressure of the lungs and airways resistance. ${ }^{10}$ As the airways resistance in SS disease has been shown to be normal, ${ }^{411}$ abnormal values for PEFR are likely to result from changes in lung compliance.

The only previous published study on PEFR in SS disease failed to show a difference between SS patients and normal controls, ${ }^{5}$ but the study was small and only four out of 12 SS patients had had previous pulmonary complications (three with one episode and one with three episodes).

The present study has shown that when populations are selected according to an absence of acute pulmonary episodes or six or more episodes, highly significant differences in PEFR between the groups emerge. In children height appears to be a major determinant of PEFR, ${ }^{6}$ but pairs were matched so that the mean difference between index cases and controls was only $0.9 \mathrm{~cm}$. It was also possible that differences in chest dimensions or shape were associated with a proneness to acute pulmonary episodes and could have contributed to the difference in PEFR. However, analysis showed that there were no significant differences between index and control cases in anteroposterior or lateral diameters of the chest, chest circumference, or anteroposterior/lateral chest diameter ratio. All dimensions correlated significantly with PEFR, the closest correlation occurring with lateral chest diameter and circumference. There was no correlation between PEFR and anteroposterior/lateral chest diameter ratio. Furthermore as the ratio did not differ between index and control cases, this suggests that the abnormal chest shape in SS disease does not result from, or predispose to, multiple episodes of acute chest syndrome.

As these physical indices of chest shape or size did not differ between the study groups, one must conclude that chronic pulmonary changes consequent on the acute chest syndrome contribute to the observed differences in PEFR. The pathological mechanism of this reduced PEFR is conjectural but parenchymal scarring presumed to result from local infarction and alveolar necrosis has been observed at necropsy. ${ }^{12}$ Such damage would be expected to be cumulative and to increase with each episode of acute chest syndrome, but there was no evidence of deteriorating PEFR with increasing episodes of six and above. It is still possible that cumulative damage would be demonstrable in patients with fewer episodes (one to five) or that the severity of the attack rather than the overall number of episodes is the major determinant of a reduced PEFR in these children.

EF Bowen was supported by grants from the Medical Research Council Rogers Award, the Royal College of Pathologists, and the British Medical and Dental Students' Trust. JG Crowsto was supported by grants from the Medical Research Council Rogers Award, and the British Medical and Dental Students' Trust. We thank Miss Joanne Morris for assistance with the statistical tests.

I De Ceulaer K, McMullen KW, Maude GH, Keatinge R, Serieant GR. Pneumonia in young children with homozygous sickle cell disease: risk and clinical features. Eur zygous sickle cell disease:

2 Miller GJ, Serjeant GR. An assessment of lung volumes and gas transfer in sickle cell anaemia. Thorax 1971;26:309-15. Miller GJ, Serjeant GR, Saunders MJ, Richardson C, Gilson RJC. Interpretation of lung function tests in the sickle-cell haemoglobinopathies. Thorax 1978;33:85-8.

4 Femi-Pearse D, Gazioglu KM, Yu PN. Pulmonary function studies in sickle cell disease. $\mathcal{F}$ Appl Physiol 1970;28:574-7. 5 Wall MA, Platt OS, Strieder DJ. Lung function in children with sickle cell anemia. Am Rev Respir Dis 1979;120:210-4 6 Godfrey S, Kamburoff PL, Nairn JR. Spirometry, lung volumes and airway resistance in normal children aged 5 to 18 years. $B r f$ Dis Chest 1970;64:15-24.

7 Herrick J. Peculiar elongation and sickle shaped red blood corpuscles in a case of severe anemia. Arch Intern Med 1910 6:517-21.

8 Barrett-Connor E. Acute pulmonary disease and sickle cell anemia. Am Rev Respir Dis 1971;104:159-65.

9 Wright BM, McKerrow CB. Maximum forced expiratory flow rate as a measure of ventilatory capacity. With a flow rate as a measure of ventilatory capacity. With a description of a new

10 Mead J, Turner J, Machler R, Little J. Significance of the relationship between lung recoil and maximum expiratory flow. F Appl Physiol 1967;22:95-108.

11 Young RC, Rachal RE, Reindorf CA, et al. Lung function in sickle cell hemoglobinopathy patients compared with healthy subjects. I Natl Med Assoc 1989;80:509-14.

12 Haupt HM, Moore GW, Bauer TW, Hutchins GM. The lung in sickle cell disease. Chest 1982;81:332-7. 